\title{
CHERN CLASSES OF INTEGRAL SUBMANIFOLDS OF SOME CONTACT MANIFOLDS
}

\author{
GHEORGHE PITIŞ
}

Received 10 March 2002

\begin{abstract}
A complex subbundle of the normal bundle to an integral submanifold of the contact distribution in a Sasakian manifold is given. The geometry of this bundle is investigated and some results concerning its Chern classes are obtained.
\end{abstract}

2000 Mathematics Subject Classification: 53C25, 57R42, 53D35.

1. Introduction. Let $\tilde{M}$ be a $(2 m+1)$-dimensional manifold endowed with the almost contact metric structure $F, \xi, \eta, g$. These tensor fields satisfy the conditions

$$
F^{2}=-I+\eta \otimes \xi, \quad \eta(\xi)=1, \quad g(F X, F Y)=g(X, Y)-\eta(X) \eta(Y),
$$

for all vector fields $X, Y$ tangent to $\tilde{M}$.

Let $\mathscr{D}$ be the contact distribution of $\tilde{M}$, defined by the equation $\eta=0$. The study of the integral submanifolds of $\mathscr{D}$ is very difficult for, at least, three reasons: (a) their abundance (see, e.g., $[1,5,8]$ ), (b) the nonexistence of a natural structure induced on the submanifold $M$, resulting from the equalities $\eta=0, d \eta=0$, true along $M$, and (c) for any vector field $X$ tangent to $M$, the vector field $F X$ is normal to $M$ and therefore, freely speaking, the geometry of an integral submanifold of $\mathscr{D}$ is normal to the submanifold. However, for maximal integral submanifolds (i.e., $\operatorname{dim} M=m$ ), we know many properties (see, e.g., [1, Chapter V]); while for nonmaximal integral submanifolds, we have so few results.

In this paper, we associate to each nonmaximal integral submanifold $M$ of $\tilde{M}$ a nontrivial vector bundle $\tau(M)$. The geometry and the topology of this vector bundle are also studied. In Section 2, we give, in an "appropriate" form, the structure equations of an integral submanifold in a Sasakian manifold. In Section 3, we study the geometry of $\tau(M)$, namely, we prove that it has a natural structure of complex symplectic vector bundle.

It is well known that integral submanifolds of an almost contact manifold are antiinvariant, [8]. Thus, such a submanifold is analogous to the isotropic (or totally real) submanifolds of a Kähler manifold, investigated by Chen and Morvan in [2, 4], and we can use some of their technics in order to study Chern classes of the vector bundle $\tau(M)$. In Section 4, by combining these ideas with some Vaisman's results [7] concerning the characteristic classes of quaternionic bundles, we obtain stronger results than for isotropic submanifolds. Namely, we prove that if $m-n$ is even, then all odd Chern classes of $\tau(M)$ are zero. In absence of this supposition on the dimensions, we prove that the first Chern class of $\tau(M)$ is zero when $\tilde{M}$ is a Sasakian space form. 
2. Structure equations of an integral submanifold. Let $\tilde{M}$ be an almost contact metric manifold. Furthermore, we assume that $\tilde{M}$ is Sasakian and let $\mathscr{L}(\tilde{M})$ denote the set of all vector fields tangent to $\tilde{M}$. We have [1, page 73]

$$
(\tilde{\nabla} F) Y=g(X, Y) \xi-\eta(Y) X, \quad X, Y \in \mathscr{X}(\tilde{M}),
$$

where $\tilde{\nabla}$ is the Levi-Civita connection associated to the metric $g$ on $\tilde{M}$. Moreover, we have the well-known equalities

$$
F \xi=0, \quad \eta \circ F=0, \quad \eta(X)=g(X, \xi), \quad \tilde{\nabla}_{X} \xi=-F X .
$$

Now, let $M$ be an $n$-dimensional submanifold of the Sasakian manifold $\tilde{M}$ and denote by $h, \tilde{\nabla}^{\perp}$, and $A$ its second fundamental form, normal connection, and Weingarten operator, respectively. It is well known that $n \leq m$ (see [8] or [1, page 36]), and we can consider in $\tilde{M}$ local fields of orthonormal frames $\mathscr{B}=\left\{e_{1}, \ldots, e_{n}, e_{n+1}, \ldots, e_{m}, e_{1 *}=\right.$ $\left.F e_{1}, \ldots, e_{n^{*}}=F e_{n}, e_{(n+1) *}=F e_{n+1}, \ldots, e_{m^{*}}=F e_{m}, e_{(m+1)} *=\xi\right\}$, with the property that the restrictions of $e_{1}, \ldots, e_{n}$ to the submanifold $M$ are tangent to $M$, so that $\mathscr{B}$ are local frames such that $T M \oplus \operatorname{span}\left\{e_{n+1}, \ldots, e_{m}\right\}$ is a Legendrian subbundle of $T \tilde{M}$.

Afterwards, we will use the following convention on the indices: $j \in\{1, \ldots, m\} ; j^{*}=$ $j+m ; a, b, c \in\{1, \ldots, n\} ; a^{*}=a+m, b^{*}=b+m, c^{*}=c+m ; \lambda, \mu, \nu \in\{n+1, \ldots, m\} ;$ $\lambda^{*}=\lambda+m ; \alpha, \beta, \gamma, \delta \in\{1, \ldots, 2 m+1\}$.

If $\mathscr{B}^{*}=\left\{\omega^{1}, \ldots, \omega^{n}, \omega^{n+1}, \ldots, \omega^{m}, \omega^{1^{*}}, \ldots, \omega^{n^{*}}, \omega^{(n+1)^{*}}, \ldots, \omega^{m^{*}}, \omega^{(m+1)^{*}}=\eta\right\}$ is the local field of coframes of $\mathscr{B}$, then, at the points of $M$, we have (locally)

$$
\omega^{\lambda}=\omega^{j^{*}}=\omega^{(m+1)^{*}}=0 .
$$

On the other hand, by computations we prove that if $\left(\omega_{\alpha}^{\beta}\right)$ is the connection form of $\tilde{\nabla}$, expressed with respect to $\mathscr{B}$, then, on the submanifold $M$, we have

$$
\begin{aligned}
\omega_{(m+1)^{*}}^{a} & =\omega_{(m+1)^{*}}^{\lambda}=\omega_{(m+1)^{*}}^{\lambda^{*}}=0, \quad \omega_{a^{*}}^{(m+1)^{*}}(X)=g\left(X, e_{a}\right), \\
\omega_{a}^{j^{*}} & =\omega_{j}^{a^{*}}, \quad \omega_{a^{*}}^{j^{*}}=\omega_{a}^{j}, \quad \omega_{\lambda}^{j^{*}}=\omega_{j}^{\lambda^{*}}, \quad \omega_{\lambda^{*}}^{j^{*}}=\omega_{\lambda}^{j} .
\end{aligned}
$$

The curvature forms of $\tilde{M}$ and $M$ are, respectively,

$$
\tilde{\Omega}_{\beta}^{\alpha}=\frac{1}{2} \sum_{\alpha, \beta=1}^{2 m+1} \tilde{R}_{\beta \gamma \delta}^{\alpha} \omega^{\gamma} \wedge \omega^{\delta}, \quad \Omega_{b}^{a}=\frac{1}{2} \sum_{c, d=1}^{n} R_{b c d}^{a} \omega^{c} \wedge \omega^{d},
$$

where $\tilde{R}_{\beta \gamma \delta}^{\alpha}$ and $R_{b c d}^{a}$ are the components (with respect to $\mathscr{B}$ ) of the curvature tensors of $\tilde{M}$ and $M$, respectively. Then, at the points of $M$, we have

$$
\begin{aligned}
& \Omega_{b}^{a}=\tilde{\Omega}_{b}^{a}-\sum_{\lambda=n+1}^{m} \omega_{\lambda}^{a} \wedge \omega_{b}^{\lambda}-\sum_{j=1}^{m} \omega_{j^{*}}^{a} \wedge \omega_{b}^{j^{*}}, \\
& \Omega_{\mu}^{\lambda}=\tilde{\Omega}_{\mu}^{\lambda}-\sum_{a=1}^{n} \omega_{a}^{\lambda} \wedge \omega_{\mu}^{a}=\frac{1}{2} \sum_{a, b=1}^{n} R_{\mu a b}^{\lambda} \omega^{a} \wedge \omega^{b},
\end{aligned}
$$

where $R_{\mu a b}^{\lambda}$ are the components of the curvature tensor of $\nabla^{\perp}$. Finally, from (2.3), (2.4), and (2.5) and from the general form of the structure equations (see, e.g., [3, page 121]), 
we deduce the structure equations of an integral submanifold of a Sasakian manifold under the form

$$
\begin{aligned}
& d \omega^{a}=-\sum_{b=1}^{n} \omega_{b}^{a} \wedge \omega^{b}, \quad d \omega_{b}^{a}=-\sum_{c=1}^{n} \omega_{c}^{a} \wedge \omega_{b}^{c}+\Omega_{b}^{a}, \\
& d \omega_{\mu}^{\lambda}=-\sum_{\nu=n+1}^{m} \omega_{\nu}^{\lambda} \wedge \omega_{\mu}^{v}-\sum_{j=1}^{m} \omega_{j^{*}}^{\lambda} \wedge \omega_{\mu}^{j^{*}}+\Omega_{\mu}^{\lambda} .
\end{aligned}
$$

3. Geometry of the maximal invariant normal bundle. The normal space $T_{x}^{\perp} M$ at each point $x \in M$ has the following orthogonal decomposition

$$
T_{x}^{\perp} M=F\left(T_{x} M\right) \oplus T_{x}(M) \oplus \operatorname{span}\left\{\xi_{x}\right\},
$$

where $\tau_{x}(M)$ is the $2(m-n)$-dimensional subspace of $T_{x} M$, orthogonal to $F\left(T_{x} M\right) \oplus$ $\operatorname{span}\left\{\xi_{x}\right\}$. Then, $\tau(M)=\bigcup_{x \in M} \tau_{x}(M)$ is the total space of a subbundle $\tau(M)$ of $T^{\perp} M$ and $\mathscr{B}_{\tau}=\left\{e_{\lambda}, e_{\lambda^{*}}\right\}=\left\{e_{n+1}, \ldots, e_{m}, e_{(n+1) *}, \ldots, e_{m} *\right\}$ is a local basis in the module $\Gamma(\tau)$ of its sections. We also denote this bundle by $\tau(M)$ and call it the maximal invariant normal bundle of the integral submanifold $M$.

THEOREM 3.1. Let $M$ be an integral submanifold of the Sasakian manifold $\tilde{M}$. Its maximal invariant normal bundle $T(M)$ has the following properties:

(a) $\tau(M)$ is invariant by $F$, that is, $F\left(\tau_{x}(M)\right)=\tau_{x}(M)$ for each $x \in M$;

(b) $\tau(M)$ has a natural structure of complex vector bundle;

(c) if $m-n=(\operatorname{dim} \tilde{M}-\operatorname{dim} M) / 2$ is even, then $\tau(M)$ has a quaternionic structure.

Proof. (a) follows easily from (3.1).

(b) Denote by $\left(n^{\lambda}, n^{\lambda^{*}}\right)$ the components of the vector $\vec{n}_{x} \in \tau_{x}(M)$, relative to the basis $\mathscr{B}_{\tau}$, and let $\rho: \tau(M) \rightarrow M$ be the natural projection. Then, using the classical notations, the vector charts

$$
\Phi: \rho^{-1}(U) \longrightarrow U \times \mathbb{C}^{m-n}, \quad \Phi\left(\vec{n}_{x}\right)=\left(x,\left(n^{\lambda}+i n^{\lambda^{*}}\right)\right), \quad x \in U,
$$

define on $\tau(M)$ a complex vector bundle structure.

(c) From (a), we deduce that the space $\Gamma(\tau)$ can be considered as a complex space with the following multiplication by complex numbers:

$$
(\alpha+i \beta) \vec{n}=\alpha \vec{n}+\beta F \vec{n}, \quad \alpha, \beta \in \mathbb{R}, \vec{n} \in \Gamma(\tau) .
$$

Endowed with this complex structure, $\Gamma(\tau)$ is an $(m-n)$-dimensional space, denoted by $\Gamma^{\mathcal{C}}(\tau)$. Moreover, we can define the map $F^{\tau}: \Gamma^{\mathcal{C}}(\tau) \rightarrow \Gamma^{\mathcal{C}}(\tau)$ by $F^{\tau}(\mathbf{n})=F \vec{n}-i F \vec{n}^{*}$ for all $\mathbf{n}=\vec{n}+i \vec{n}^{*}, \vec{n}, \vec{n}^{*} \in \Gamma(\tau)$, and it has the following properties:

$$
F^{\mathcal{T}}\left(\mathbf{n}_{1}+\mathbf{n}_{2}\right)=F^{\tau} \mathbf{n}_{1}+F^{\mathcal{T}} \mathbf{n}_{2}, \quad F^{\mathcal{T}}(\lambda \mathbf{n})=\bar{\lambda} F^{\mathcal{T}} \mathbf{n}, \quad\left(F^{\mathcal{T}}\right)^{2} \mathbf{n}=-\mathbf{n},
$$

for $\mathbf{n}, \mathbf{n}_{1}, \mathbf{n}_{2} \in \Gamma^{\mathcal{c}}(\tau)$ and $\lambda \in \mathbb{C}$. Hence (see [7, Section 1]), $F^{\tau}$ defines on $\boldsymbol{\tau}(M)$ a quaternionic structure. 
A natural connection can be defined on $\tau(M)$. Firstly, we remark that $g\left(\nabla_{X}^{\frac{1}{X}} \vec{n}, \xi\right)=0$ for all $X \in \mathscr{X}(M)$ and $\vec{n} \in \Gamma(\tau)$, hence the normal vector field $\nabla_{X}^{\frac{1}{n}} \vec{n}$ has the following decomposition:

$$
\nabla_{X}^{\perp} \vec{n}=B_{\vec{n}} X+\nabla_{X}^{\tau} \vec{n}
$$

where $B_{\vec{n}} X \in \Gamma(F T M)$ and $\nabla_{X}^{\tau} \vec{n} \in \Gamma(\tau)$. Moreover, the maps $B: \Gamma(\tau) \times \mathscr{L}(M) \rightarrow \Gamma(F T M)$ and $\nabla^{\tau}: \mathscr{X}(M) \times \Gamma(\tau) \rightarrow \Gamma(\tau)$ have the following properties.

Proposition 3.2. (a) $\nabla^{\top}$ is an almost complex connection on the maximal invariant normal bundle of the integral submanifold $M$, that is, $\left(\nabla_{X}^{\tau} F\right) \vec{n}=0$.

(b) $B_{\vec{n}} X=F A_{F \vec{n}} X$ for all $X \in \mathscr{L}(M)$ and $\vec{n} \in \Gamma(\tau)$.

The proof follows from (3.5) by computation, taking into account (2.1) and (2.2) and using the Weingarten formula for the submanifold $M$.

Now, if we extend the scalar product $g$ over $\Gamma^{c}(\tau)$ by

$$
g^{\top}\left(\mathbf{n}_{1}, \lambda \mathbf{n}_{2}\right)=\bar{\lambda} g^{\top}\left(\mathbf{n}_{1}, \mathbf{n}_{2}\right), \quad g^{\top}\left(\mathbf{n}_{2}, \mathbf{n}_{1}\right)=\overline{g^{\top}\left(\mathbf{n}_{1}, \mathbf{n}_{2}\right)},
$$

for $\lambda \in \mathbb{C}$ and $\mathbf{n}_{1}, \mathbf{n}_{2} \in \Gamma^{\mathcal{C}}(\tau)$, then we have

$$
g^{\top}\left(F^{\top} \mathbf{n}_{1}, F^{\top} \mathbf{n}_{2}\right)=\overline{g^{\top}\left(\mathbf{n}_{1}, \mathbf{n}_{2}\right)},
$$

hence $g^{\top}$ is a Hermitian scalar product on the complex vector bundle $\tau(M)$. Moreover, $\mathscr{B}_{\tau}^{c}=\left\{f_{\lambda}=(1 / \sqrt{2})\left(e_{\lambda}+i e_{\lambda^{*}}\right), f_{\lambda^{*}}=(1 / \sqrt{2})\left(e_{\lambda}-i e_{\lambda^{*}}\right)\right\}$ is an orthonormal local basis of $\Gamma^{c}(\tau)$ with respect to $g^{\tau}$ and $f_{\lambda^{*}}=-i f_{\lambda}, F^{\tau} f_{\lambda}=i \overline{f_{\lambda}}=f_{\lambda^{*}}$.

For any $\mathbf{n}_{1}, \mathbf{n}_{2} \in \Gamma^{c}(\tau)$, we put

$$
\Omega^{\top}\left(\mathbf{n}_{1}, \mathbf{n}_{2}\right)=-\overline{g^{\top}\left(F^{\top} \mathbf{n}_{1}, \mathbf{n}_{2}\right)},
$$

and a simple computation shows that $\Omega^{\top}$ is $\mathbb{C}$-linear with respect to the first argument and

$$
\Omega^{\top}\left(\mathbf{n}_{1}, \mathbf{n}_{2}\right)=-\overline{\Omega^{\top}\left(\mathbf{n}_{2}, \mathbf{n}_{1}\right)}, \quad \Omega^{\top}\left(F^{\top} \mathbf{n}_{1}, F^{\top} \mathbf{n}_{2}\right)=\overline{\Omega^{\top}\left(\mathbf{n}_{1}, \mathbf{n}_{2}\right)} .
$$

From these relations and because $\mathscr{B}_{\tau}^{c}$ is an orthonormal local basis, we deduce that $\Omega^{T}$ is a nondegenerate skew-symmetric 2 -form on the complex vector bundle $\tau(M)$. Hence, we have the following proposition.

Proposition 3.3. For $m-n$ even, the maximal invariant normal bundle $\tau(M)$ of the integral submanifold $M$ of a Sasakian manifold has a structure of complex symplectic vector bundle with the symplectic form $\Omega^{T}$.

4. Normal Chern classes of an integral submanifold. As a complex vector bundle, the basic characteristic classes of the maximal invariant normal bundle $\tau(M)$ are the Chern classes $\left[\gamma_{k}(\tau)\right]$, represented by the Chern forms

$$
\gamma_{k}=\frac{i^{k}}{(2 \pi)^{k} k !} \delta_{\lambda_{1} \cdots \lambda_{k}}^{\mu_{1} \cdots \mu_{k}} \stackrel{\tau^{\lambda_{1}}}{\lambda_{\mu_{1}}} \wedge \cdots \wedge \stackrel{\tau^{\lambda_{k}}}{\lambda_{\mu_{k}}},
$$


where $\Omega_{\mu}^{\tau_{\mu}^{\lambda}}$ are the curvature forms of $\nabla^{\tau}$ and $\delta \cdots$ is the multiindex Kronecker symbol. We say that $\gamma_{k}(\tau)$ is the $k$ th normal Chern form of the submanifold $M$ and the purpose of this section is to obtain some results concerning the computation of $\gamma_{k}(\tau)$ and the $k$ th normal Chern class $\left[\gamma_{k}(\tau)\right]$ of $M$.

THEOREM 4.1. Let $M$ be an $n$-dimensional integral submanifold of a Sasakian manifold of dimension $2 m+1$. If $m-n$ is even, then

$$
\left[\gamma_{2 k+1}(\tau)\right]=0 \quad \text { for } k=0,1, \ldots,\left[\frac{m-n-1}{2}\right] .
$$

Proof. By Theorem 3.1(c), the maximal invariant normal bundle $\tau(M)$ has a quaternionic structure, and then we can apply [7, Proposition 2.1].

Now, we will analyse the first normal Chern form and its associated class in absence of the supposition that $m-n$ is even.

THEOREM 4.2. The first normal Chern form of the n-dimensional integral submanifold $M$ in a Sasakian manifold of dimension $2 m+1, m>n$, is given by

$$
\gamma_{1}(\tau)=\frac{1}{2 \pi} \sum_{\lambda=n+1}^{m} \Omega_{\lambda}^{\lambda^{*}}
$$

Proof. Using (3.5), (2.1), and the Weingarten formula, we obtain the components of the curvature tensor $\stackrel{T}{R}$ of $\nabla^{\top}$ under the form

$$
\stackrel{\tau^{\lambda^{*}}}{R_{\lambda a b}}=R_{\lambda a b}^{\lambda^{*}}+g\left(B_{e_{\lambda}} e_{b}, B_{e_{\lambda^{*}}} e_{a}\right)-g\left(B_{e_{\lambda}} e_{a}, B_{e_{\lambda^{*}}} e_{b}\right),
$$

and then its curvature form is $\stackrel{\tau_{\lambda}^{\lambda^{*}}}{\Omega_{\lambda}}=\Omega_{\lambda}^{\lambda^{*}}$. On the other hand, from (2.9), it follows the complex form of the second structure equation of $\tau(M)$, namely,

$$
\begin{gathered}
d \phi_{\mu}^{\lambda}=-\sum_{\nu=n+1}^{m} \phi_{\nu}^{\lambda} \wedge \phi_{\mu}^{\nu}+\Phi_{\mu}^{\lambda} \quad \text { with } \phi^{\lambda}=\omega^{\lambda}+i \omega^{\lambda^{*}}, \\
\phi_{\mu}^{\lambda}=\omega_{\mu}^{\lambda}+i \omega_{\mu^{*}}^{\lambda}, \quad \Phi_{\mu}^{\lambda}=\stackrel{\tau^{\lambda}}{\Omega_{\mu}}+i \stackrel{\tau^{\lambda}}{\Omega_{\mu^{*}}} .
\end{gathered}
$$

But $\Phi_{\lambda}^{\lambda}=i{\stackrel{\tau}{\Omega_{\lambda}} \lambda^{\lambda}}$, and then we have

$$
\gamma_{1}(\tau)=\frac{i}{2 \pi} \sum_{\lambda=n+1}^{m} \Phi_{\lambda}^{\lambda}=-\frac{1}{2 \pi} \sum_{\lambda=n+1}^{m} \stackrel{\tau^{\lambda}}{\Omega_{\lambda *}}=-\frac{1}{2 \pi} \sum_{\lambda=n+1}^{m} \Omega_{\lambda *}^{\lambda}
$$

and the proof is complete.

Let $\vec{n}$ be a vector field normal to the integral submanifold $M$ of the Sasakian manifold $\tilde{M}$. For $X \in \mathscr{L}(M)$, the equality $\alpha_{\vec{n}}(X)=g(F \vec{n}, X)$ defines a 1 -form $\alpha_{\vec{n}}$ on $M$. In [6], this form is used for the study of some remarkable vector fields on $M$ (Legendrian, Hamiltonian, and harmonic variations). Another 1-form on $M$ is defined by $\theta=\sum_{a=1}^{n} \omega_{a}^{a^{*}}$, and we can state the following proposition. 
Proposition 4.3. The forms $\alpha_{\vec{n}}$ and $\theta$ have the following properties:

(a) $\alpha_{\xi}=0$ and $\theta=-n \alpha_{H}$, where $H$ is the mean curvature vector of $M$;

(b) $\alpha_{\vec{n}}$ is closed if and only if

$$
g\left(\nabla_{X}^{\frac{1}{n}} \vec{n}, F Y\right)=g\left(\nabla_{Y}^{\frac{1}{n}} \vec{n}, F X\right)
$$

for all $X, Y \in \mathscr{X}(M)$;

(c) the exterior derivative of $\theta$ is given by

$$
d \theta=\sum_{b, c=1}^{n}\left(\tilde{S}_{b c^{*}}-\sum_{\lambda} R_{\lambda b c}^{\lambda^{*}}-\frac{1}{2} \sum_{a=1}^{n} \tilde{R}_{a b c}^{a^{*}}\right) \omega^{b} \wedge \omega^{c},
$$

where $\tilde{S}$ is the Ricci tensor of $\tilde{M}$.

Proof. (a) We have the well-known equality

$$
\tilde{\nabla}_{X} e_{\alpha}=\sum_{\beta=1}^{m} \omega_{\alpha}^{\beta}(X) e_{\beta}
$$

for any $X \in \mathscr{L}(\tilde{M})$, and, by using (2.4) and (2.5), we obtain

$$
\theta\left(e_{b}\right)=\sum_{a=1}^{n} g\left(\tilde{\nabla}_{e_{b}} e_{a}, e_{a^{*}}\right), \quad b \in\{1,2, \ldots, n\} .
$$

Taking into account (2.1) and the Gauss formula, we deduce

$$
\begin{aligned}
\theta\left(e_{b}\right) & =\sum_{a=1}^{n} g\left(h\left(e_{a}, e_{b}\right), e_{a^{*}}\right)=\sum_{a=1}^{n} g\left(\tilde{\nabla}_{e_{a}} e_{b}, F e_{a}\right) \\
& =-\sum_{a=1}^{n} g\left(F\left(\tilde{\nabla}_{e_{a}} e_{b}\right), e_{a}\right)=-\sum_{a=1}^{n} g\left(\tilde{\nabla}_{e_{a}} e_{b^{*}}, e_{a}\right)=\sum_{a=1}^{n} g\left(e_{b^{*}}, \tilde{\nabla}_{e_{a}} e_{a}\right) \\
& =\sum_{a=1}^{n} g\left(e_{b^{*}}, h\left(e_{a}, e_{a}\right)\right)=n g\left(e_{b^{*}}, H\right)=-n g\left(e_{b}, F H\right)=-n \alpha_{H}\left(e_{b}\right) .
\end{aligned}
$$

(b) From the definition of the 1 -form $\alpha_{\vec{n}}$, we obtain

$$
d \alpha_{\vec{n}}(X, Y)=g\left(\tilde{\nabla}_{X}(F \vec{n}), Y\right)-g\left(\tilde{\nabla}_{Y}(F \vec{n}), X\right) .
$$

On the other hand, using (2.1), we have

$$
\tilde{\nabla}_{X}(F \vec{n})=F\left(\tilde{\nabla}_{X} \vec{n}\right)-\eta(\vec{n}) X,
$$

and then, applying the Weingarten formula in (4.12), it follows that

$$
d \alpha_{\vec{n}}(X, Y)=g\left(\tilde{\nabla}_{Y}^{\perp} \vec{n}, F X\right)-g\left(\tilde{\nabla}_{X}^{\frac{1}{n}} \vec{n}, F Y\right) .
$$

(c) From (2.4) and (2.5) and taking into account the second structure equation of $\tilde{M}$

$$
d \omega_{\beta}^{\alpha}=-\sum_{\gamma=1}^{2 m} \omega_{\gamma}^{\alpha} \wedge \omega_{\beta}^{\gamma}+\tilde{\Omega}_{\beta}^{\alpha},
$$


we obtain

$$
\begin{aligned}
d \theta= & -\sum_{\lambda=n+1}^{m} \sum_{a=1}^{n} \omega_{\lambda}^{a^{*}} \wedge \omega_{a}^{\lambda}-\sum_{b=1}^{n} \sum_{a=1}^{n} \omega_{b}^{a} \wedge \omega_{a}^{b^{*}} \\
& -\sum_{\lambda=n+1}^{m} \sum_{a=1}^{n} \omega_{\lambda}^{a} \wedge \omega_{a}^{\lambda^{*}}+\sum_{a=1}^{n} \tilde{\Omega}_{a}^{a^{*}} .
\end{aligned}
$$

Using (2.5) again, the above equality becomes

$$
d \theta=2 \sum_{a=1}^{n} \sum_{\lambda=n+1}^{m} \omega_{a}^{\lambda} \wedge \omega_{a}^{\lambda^{*}}+\sum_{a=1}^{n} \tilde{\Omega}_{a}^{a^{*}}
$$

Now, applying the Gauss formula for the submanifold $M$ in (4.9), we have

$$
\sum_{b=1}^{n}\left[\omega_{a}^{b}(X) e_{b}+\omega_{a}^{b^{*}}(X) e_{b^{*}}\right]+\sum_{\lambda=n+1}^{m}\left[\omega_{a}^{\lambda}(X) e_{\lambda}+\omega_{a}^{\lambda^{*}}(X) e_{\lambda^{*}}\right]=\nabla_{X} e_{a}+h\left(X, e_{a}\right)
$$

for any $X \in \mathscr{X}(M)$. It follows that

$$
\begin{aligned}
\omega_{a}^{\mu}(X) & =g\left(h\left(X, e_{a}\right), e_{\mu}\right) \\
& =\sum_{b=1}^{n} X^{b} g\left(h\left(e_{a}, e_{b}\right), e_{\mu}\right)=\sum_{b=1}^{n} X^{b} h_{b a}^{\mu}=\sum_{b=1}^{n} h_{b a}^{\mu} \omega^{b}(X),
\end{aligned}
$$

where $h_{a c}^{\alpha}$ are the components of $h\left(e_{a}, e_{c}\right)$ with respect to the basis $\mathscr{B}_{\tau}$. Therefore, we have

$$
\omega_{a}^{\alpha}=\sum_{a=1}^{n} h_{a c}^{\alpha} \omega^{c}
$$

for any $\alpha=\lambda$ or $\alpha=\lambda^{*}$. Finally, from (4.17) and (4.20) we deduce

$$
d \theta=\sum_{a, b, c=1}^{n}\left(h_{a b}^{\lambda} h_{a c}^{\lambda^{*}}-h_{a c}^{\lambda} h_{a b}^{\lambda^{*}}\right) \omega^{b} \wedge \omega^{c}+\sum_{a=1}^{n} \tilde{\Omega}_{a}^{a^{*}} .
$$

Because $\tilde{M}$ is Sasakian, its curvature tensor $\tilde{R}$ satisfies the following equality [1, page 75]:

$$
\tilde{R}(X, Y) \xi=\eta(Y) X-\eta(X) Y, \quad X, Y \in \mathscr{X}(\tilde{M}),
$$

hence the Ricci tensor $\tilde{S}$ of $\tilde{M}$ is given by

$$
\tilde{S}(X, Y)=\sum_{\alpha=1}^{2 m} \tilde{\mathscr{R}}\left(e_{\alpha}, X, e_{\alpha}, Y\right)-g(X, Y),
$$

for all $X, Y \in \mathscr{L}(\tilde{M})$ orthogonal to $\xi$, where $\tilde{\mathscr{R}}$ is the Riemann-Christoffel curvature tensor field of $\tilde{M}$. 
Using (2.3), from the first equality in (2.6), we deduce

$$
\sum_{a=1}^{n} \tilde{\Omega}_{a}^{a^{*}}=\frac{1}{2} \sum_{a, b, c=1}^{n} \tilde{R}_{a b c}^{a^{*}} \omega^{b} \wedge \omega^{c}
$$

at any point of the submanifold $M$. Moreover, using the first Bianchi identity relative to $\tilde{M}$, we have

$$
\tilde{R}_{a b c}^{a^{*}}=\tilde{\mathscr{R}}\left(e_{a^{*}}, e_{a}, e_{b}, e_{c}\right)=\tilde{\mathscr{R}}\left(e_{c}, e_{a^{*}}, e_{a}, e_{b}\right)+\tilde{\mathscr{R}}\left(e_{c}, e_{a}, e_{b}, e_{c^{*}}\right) .
$$

On the other hand, on a Sasakian manifold, the following equalities are true [1, page 93]:

$$
\begin{aligned}
\tilde{\mathscr{R}}(F X, F Y, F Z, F U)= & \tilde{\mathscr{R}}(X, Y, Z, U), \\
\tilde{\mathscr{R}}(F X, Y, Z, U)+\tilde{\mathscr{R}}(X, F Y, Z, U)= & d \eta(Y, Z) g(U, X)+d \eta(Z, X) g(Y, U) \\
& +d \eta(U, Y) g(X, Z)+d \eta(X, U) g(Y, Z),
\end{aligned}
$$

for all $X, Y, Z, U \in \mathscr{L}(\tilde{M})$ orthogonal to $\xi$. But $d \eta\left(e_{a}, e_{b}\right)=0$, hence, from (4.27), we deduce

$$
\tilde{\mathscr{R}}\left(e_{a^{*}}, e_{c}, e_{a}, e_{b}\right)+\tilde{\mathscr{R}}\left(e_{a}, e_{c^{*}}, e_{a}, e_{b}\right)=0
$$

and therefore, using (4.23) and (4.26), from (4.25) we obtain

$$
\begin{aligned}
\sum_{a=1}^{n} \tilde{R}_{a b c}^{a^{*}} & =\sum_{a=1}^{n}\left[\tilde{\mathscr{R}}\left(e_{a}, e_{b}, e_{a}, e_{c^{*}}\right)+\tilde{\mathscr{R}}\left(e_{a^{*}}, e_{b}, e_{a^{*}}, e_{c^{*}}\right)\right] \\
& =\tilde{S}\left(e_{b}, e_{c^{*}}\right)+\sum_{\lambda=n+1}^{m}\left[\tilde{\mathscr{R}}\left(e_{c^{*}}, e_{\lambda}, e_{\lambda}, e_{b}\right)+\tilde{\mathscr{R}}\left(e_{\lambda}, e_{b^{*}}, e_{\lambda}, e_{c}\right)\right] .
\end{aligned}
$$

Now, from (4.27), we give

$$
\tilde{\mathscr{R}}\left(e_{a^{*}}, e_{\lambda}, e_{\lambda}, e_{b}\right)+\tilde{\mathscr{R}}\left(e_{a}, e_{\lambda *,} e_{\lambda}, e_{b}\right)=0
$$

and then

$$
\sum_{a=1}^{n} \tilde{R}_{a b c}^{a^{*}}=\tilde{S}\left(e_{b}, e_{c^{*}}\right)+\sum_{\lambda=n+1}^{m}\left[\tilde{\mathscr{R}}\left(e_{\lambda^{*}}, e_{c}, e_{\lambda}, e_{b}\right)+\tilde{\mathscr{R}}\left(e_{\lambda^{*}}, e_{b}, e_{c}, e_{\lambda}\right)\right] .
$$

Applying the second Bianchi identity in the above equality, we obtain

$$
\sum_{a=1}^{n} \tilde{R}_{a b c}^{a^{*}}=\tilde{S}\left(e_{b}, e_{c^{*}}\right)-\sum_{\lambda=n+1}^{m} \tilde{\mathscr{R}}\left(e_{\lambda^{*}}, e_{\lambda}, e_{b}, e_{c}\right)
$$

and taking into account the Ricci equation

$$
\tilde{\mathscr{R}}\left(e_{\lambda^{*}}, e_{\lambda}, e_{b}, e_{c}\right)=\mathscr{R}^{\perp}\left(e_{\lambda^{*}}, e_{\lambda}, e_{b}, e_{c}\right)-g\left(\left[A_{e_{\lambda^{*}}}, A_{e_{\lambda}}\right] e_{c}, e_{b}\right),
$$


we deduce

$$
\sum_{a=1}^{n} \tilde{R}_{a b c}^{a^{*}}=\tilde{S}_{b c^{*}}-\sum_{\lambda=n+1}^{m} R_{\lambda b c}^{\lambda^{*}}+\sum_{\lambda=n+1}^{m} \sum_{d=1}^{n}\left(A_{\lambda c}^{d} A_{\lambda * d}^{b}-A_{\lambda{ }^{*} c}^{d} A_{\lambda d}^{b}\right),
$$

where, by $A_{\lambda a}^{b}$, we denote the components of the Weingarten operator of $M$, relative to $\mathscr{B}$. Now, (4.8) follows from (4.17), (4.34), and (2.6).

THEOREM 4.4. Let $M$ be an integral submanifold of the Sasakian space form $\tilde{M}(c)$.

(a) The first normal Chern class $\left[\gamma_{1}(\tau)\right]$ of $M$ is zero.

(b) If the mean curvature vector of $M$ is parallel, then its first normal Chern form $\gamma_{1}(\tau)$ is zero.

PROoF. (a) Recall that in a Sasakian space form $\tilde{M}(c)$, the curvature tensor $\tilde{R}$ and the Ricci tensor $\tilde{S}$ have the following expressions (see, e.g., [1, pages, 97-98]):

$$
\begin{aligned}
\tilde{R}(X, Y) Z= & \frac{c+3}{4}[g(Y, Z) X-g(X, Z) Y] \\
& +\frac{c-1}{4}[\eta(X) \eta(Z) Y-\eta(Y) \eta(Z) X+g(X, Z) \eta(Y) \xi-g(Y, Z) \eta(X) \xi \\
& \quad+g(Z, F Y) F X-g(Z, F X) F Y-2 g(X, F Y) F Z], \\
\tilde{S}(X, Y)= & \frac{m(c+3)+c-1}{2} g(X, Y)-\frac{(m+1)(c-1)}{2} \eta(X) \eta(Y),
\end{aligned}
$$

for all $X, Y, Z \in \mathscr{L}(\tilde{M})$. From these equalities, we easily deduce $\tilde{R}_{a b c}^{a^{*}}=0, \tilde{S}_{b c^{*}}=0$, and taking into account (2.8) from Proposition 4.3(c), we obtain

$$
d \theta=-2 \sum_{\lambda=n+1}^{m} \Omega_{\lambda}^{\lambda^{*}}
$$

From Theorem 4.2 and from Proposition 4.3(a) and (c), it follows that

$$
d \alpha_{H}=-\frac{1}{n} d \theta=\frac{4 \pi}{n} \gamma_{1}(\tau)
$$

and then the assertion (a) is proved.

(b) From (4.36) and using Proposition 4.3(b), we obtain $\gamma_{1}(\tau)=0$.

\section{REFERENCES}

[1] D. E. Blair, Contact Manifolds in Riemannian Geometry, Lecture Notes in Mathematics, vol. 509, Springer-Verlag, Berlin, 1976.

[2] B.-Y. Chen and J.-M. Morvan, Deformations of isotropic submanifolds in Kähler manifolds, J. Geom. Phys. 13 (1994), no. 1, 79-104.

[3] S. Kobayashi and K. Nomizu, Foundations of Differential Geometry. Vol I, Interscience Publishers, New York, 1963.

[4] J.-M. Morvan, Classes caractéristiques des sous-variétés isotropes [Characteristic classes of isotropic submanifolds], C. R. Acad. Sci. Paris Sér. I Math. 308 (1989), no. 9, 269-272 (French).

[5] Gh. Pitiş, On parallel submanifolds of a Sasakian space form, Rend. Mat. Appl. (7) 9 (1989), no. $1,103-111$. 
[6] _ Stability of integral submanifolds in a Sasakian manifold, Kyungpook Math. J. 41 (2001), no. 2, 381-392.

[7] I. Vaisman, Exotic characteristic classes of quaternionic bundles, Israel J. Math. 69 (1990), no. $1,46-58$.

[8] K. Yano and M. Kon, Anti-invariant submanifolds of Sasakian space forms. II, J. Korean Math. Soc. 13 (1976), no. 1, 1-14.

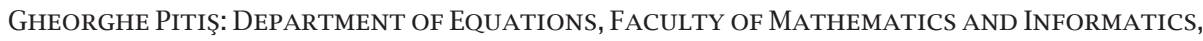
TRANSILVANIA UNIVERSITY OF BRAŞOV, ROMANIA

E-mail address: gh.pitis@info.unitbv.ro 


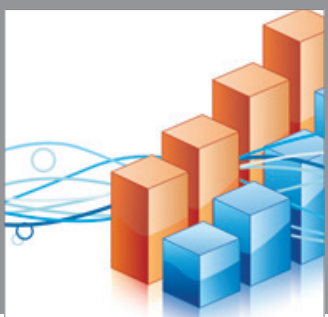

Advances in

Operations Research

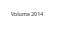

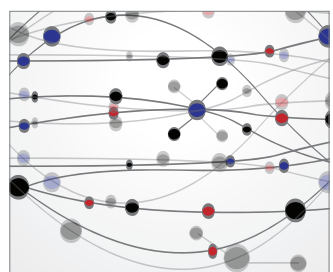

\section{The Scientific} World Journal
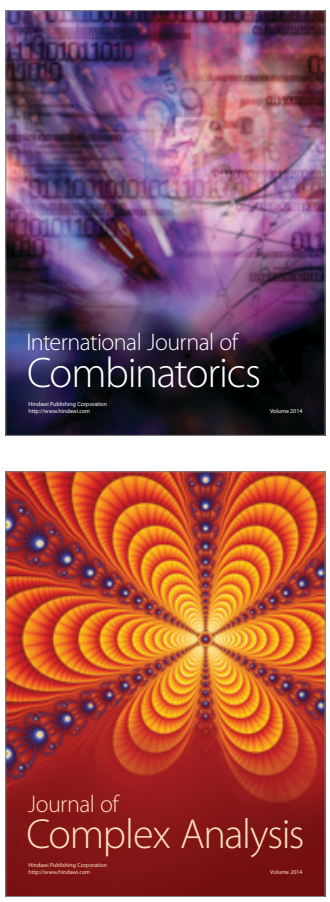

International Journal of

Mathematics and

Mathematical

Sciences
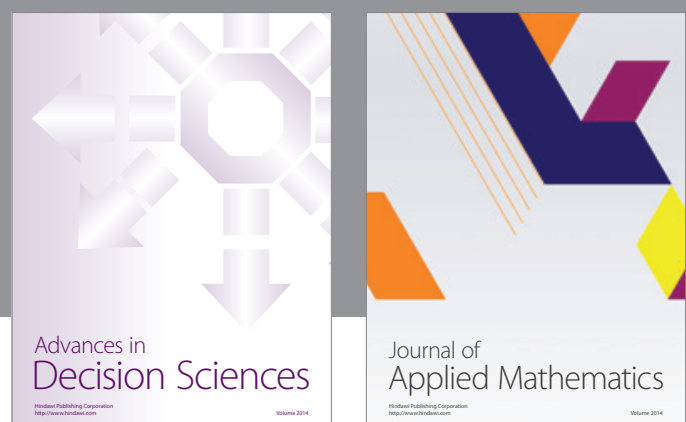

Journal of

Applied Mathematics
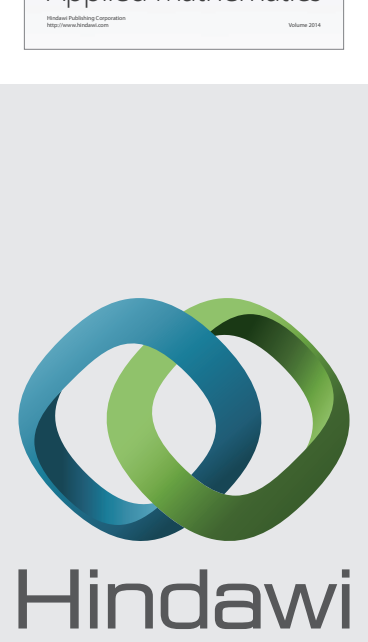

Submit your manuscripts at http://www.hindawi.com
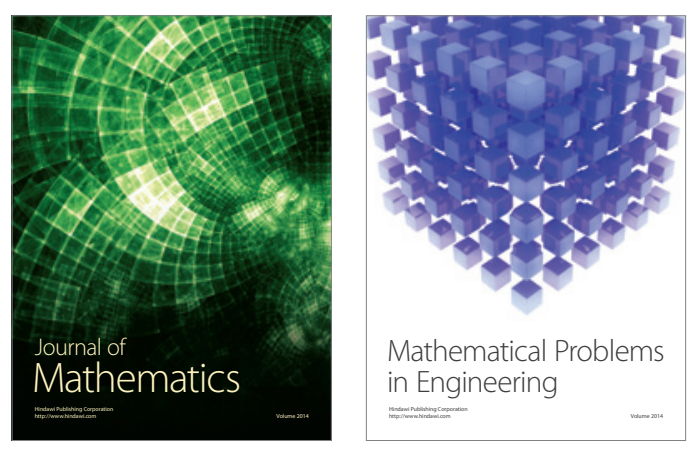

Mathematical Problems in Engineering
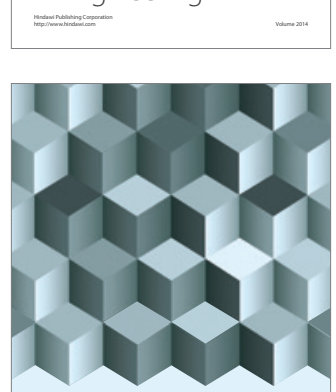

Journal of

Function Spaces
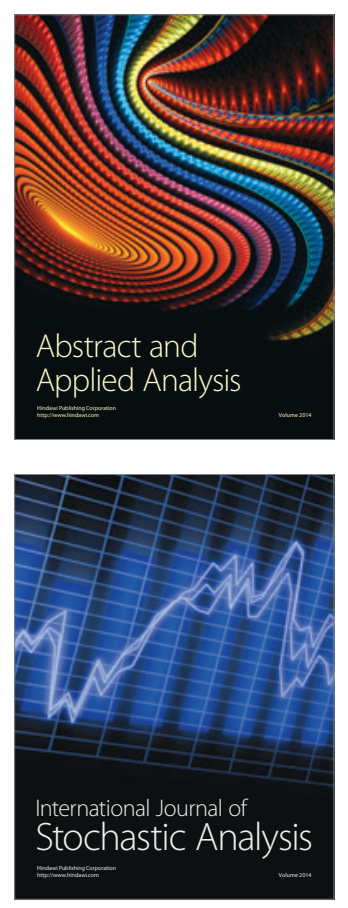

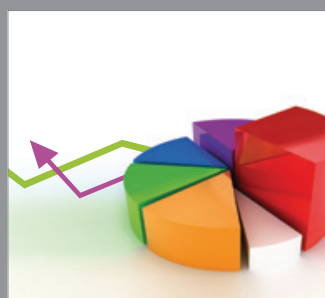

ournal of

Probability and Statistics

Promensencen
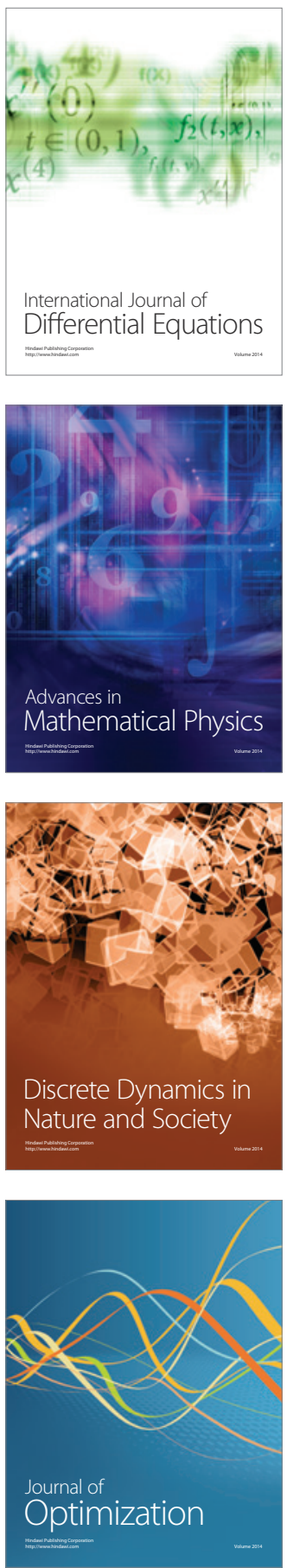